\title{
The stethoscope goes digital: Learning through attention, distraction and distortion*
}

\author{
Melissa Van Drie and Anna Harris
}

\begin{abstract}
For centuries, those training doctors have been faced with the challenges of standardising subjective experiences and constructing "the universal body" in learning situations. Various technologies have been introduced to address these challenges, with varying degrees of success. In this article we focus on the stethoscope, specifically the electrical and digital stethoscope models. Historical and social studies of medicine have already underlined the sociomateriality of learning in medicine. In this article we underscore the per-

* Acknowledgements: This article draws on research funded by a Dutch NWO Vici Grant entitled Sonic Skills: Sound and Listening in the Development of Science, Technology, Medicine (1920-now) awarded to Karin Bijsterveld (grant agreement No: 277-45-003).

Melissa Van Drie further developed the article's historiographical approaches to sensory experiences of sound devices and did archival research on hearing disabilities during a postdoctoral position in the European Research Council (ERC) funded project Sound and Materialism in the $19^{\text {th }}$ Century awarded to David Trippett at the University of Cambridge (Horizon 2020 scheme, grant agreement No. 638241). Final corrections were completed during Melissa's current ERC funded Marie Skłodowska-Curie Individual Fellowship project called Sounds Delicious at the University of Copenhagen (grant agreement No. 753565).

The article also draws on Anna Harris' current research on digital doctors funded by the European Research Council (ERC) under the European Union's Horizon 2020 research and innovation program (grant agreement No. 678390).

Anna would like to thank the participants in her studies for their time and for sharing their experiences, skills and stethoscopes with her, and Ruth Benschop for introducing Hirschauer's work. Melissa and Anna would like to thank the Sonic Skills team for their constructive feedback on our collaborative project along the way, as well as Whitney Laemmli, Kate Smith, Josh Grace, Caitlin Wylie, Johanna Gonçalves Martin, Stewart Allen, Nina Lerman and Dagmar Schäfer for their comments on earlier drafts during our time together at the Max Planck Institute for the History of Science in Berlin. Finally, Melissa and Anna are grateful to the anonymous reviewers, and to Thibault Walter and Vincent Barras for encouraging them to develop a conference presentation into this piece, and for their support along the way.
\end{abstract}

Melissa Van Drie, Department of Arts and Cultural Studies, University of Copenhagen, Karen Blixens vej 1,2300 Copenhagen, DK, vandrie@hum.ku.dk

Anna Harris, Department of Technology Studies, Faculty of Arts and Social Sciences, Maastricht University, Postbus/ P.O. Box 616, 6200 MD Maastricht, NL, a.harris@maastrichtuniversity.nl 
formative nature of teaching and learning in the sociomaterial context. We do so by juxtaposing ethnographic and historical events that stage electrical and digital stethoscopes. These are not documentations of everyday practices but rather reconstructions of choreographed performances for learning about the body. In these stagings, the novice is taught to focus attention and avoid distraction, when learning the sounds of "the body". Through engaging with, and comparing, different ethnographic and historic materials and artefacts, and through methodological reflection, we examine the importance not only of attention and distraction in learning a bodily skill, but also of dealing with distortion. We argue that these ethnographic and historic insights into distortion illuminate a neglected aspect of medical training, and more generally, in shaping sensory perceptions.

Keywords: digital stethoscope, electrical stethoscope, medical training, listening, auscultation, sensory studies

\section{Introduction}

The training of medical doctors involves not only an individual embodied process of enskillment, ${ }^{1}$ but also learning about other bodies too; how bodies sound, smell, feel and appear, both in their "normal" and "pathological" states.

For centuries medical educators have been faced with the challenge of standardising these sensory subjective experiences and constructing "the universal body" in learning situations. Undeniably, every practitioner experiences these sensations differently. Various techniques and technologies have been introduced to address these challenges in order to train doctors to see, listen and touch, with varying degrees of success. Examples include: the two-headed microscope; ${ }^{2}$ simulation mannequins; and teaching videos and interactive websites. ${ }^{3}$

In this article we focus on a technology common in medical practice, the stethoscope, specifically the electronic and digital stethoscope models - which were, and are, used in medical teaching to educate students on how and what body sounds to listen to. Our paper engages with, and compares, different ethnographic and historical materials and artefacts concerning how medical students have learnt to listen to sounds from the $19^{\text {th }}$ century until today.

In this paper that is dedicated to situations of learning bodily practices, the sociomaterial contexts involved in staging such learning (including the modes

1 Ingold 2000.

2 Atkinson 1995.

3 Prentice 2005; Johnson 2007. 
of framing information) are important. Elaborating on our own research approaches, sites and conditions is also a part of this endeavour. Melissa Van Drie's historical inquiry happened first between 2011 and 2012, when she started searching for stories and artefacts on how novices were taught the art of auscultation, or how to listen to bodies. Working in British, American and French medical archives, ${ }^{4}$ she compared all kinds of pedagogical resources (dating from 1850 to 2010) and the links between them, such as textbooks, audio and audio-visual materials, photographs, medical device catalogues and technical journals. ${ }^{5}$ In 2016, Melissa returned to the topic of performances of medical knowledge, noticing the use of electronic stethoscopes and sound amplification in live situations of collective learning. In 2013, Anna Harris spent five months as participant observer in a medical school and teaching hospital in Melbourne, Australia, and one month in a medical school in Maastricht, The Netherlands. During fieldwork, she followed the ways in which medical students were taught and learned how to listen to sounds of the body, specifically respiratory sounds. She followed this up with further fieldwork in 20172018 , looking at how medical students learn to sense the body more generally. ${ }^{6}$

Our method in this paper is one of a juxtaposition of events: ethnographic and historical events. We define event here, following Karen Barad, as a performative and dynamic intra-action of objects, media, bodies, ideas, ephemera and sensations that makes one believe that something has happened and through which we can understand something about what is at stake in our enquiry. These events are not "stable" but rather held still for a moment as we consider them. The ethnographic events are crafted from fieldnotes, but rather than constructing them as documentations of an actual occurrence, it is the crafted re-telling of what was observed and experienced during fieldwork that becomes the event presented here. The historical events reconstitute the sonic event and performance of the technique through different modes of engaging artefacts. Centered on the listening apparatus of the 'stethophone', the first electronic stethoscope used as a teaching device, the historical events use visual traces from 1924 as a starting point: a photograph in the British Hospital and Health Journal shows medical students listening to a patient in hospital; ${ }^{8}$ and a schematic drawing published in the Bell Sys-

4 Archives included: Wellcome Collection and British Library (London, UK), US National Library of Medicine (Bethesda, Maryland, USA) and Library of Congress (Washington D.C., USA), Bibliothèque Interuniversitaire de Santé, Bibliothèque de l'Académie nationale de Médicine, Bibliothèque nationale de France (Paris, FR).

5 Van Drie 2013.

6 See also Harris/Van Drie 2015; Harris 2016.

7 Barad 2007.

8 "Heartbeats by Radio" 1924. 
tem Technical Journal depicts the installation of the electrical wiring of the lecture hall and hospital ward. ${ }^{9}$ Within their initial contexts, both images accompany text about a technological system. Yet considered as representations of sensory modes and experiences, and considered within frames of performance, they incite a different material culture reading. Here, the hermeneutic process of the historian's profession is palpable through how affinities are drawn between different textual, visual, sonic information found in scientific research, technological trade journals, medical textbooks, photographs, sound recordings. ${ }^{10}$ Additionally, acts of "deep-description" of such historical documents correspond to the ethnographic field notes as we seek to reflect on our disciplinary ways of shaping boundaries and perspectives. ${ }^{11}$

Through this juxtaposition of different mediatised, wired, digitised events of stethoscopic listening we explore an element of learning practices that is often neglected. While we attend to the aspects of attention and distraction, which others have underlined as necessary to harness in order to educate the senses, we focus here on the less discussed issue of distortion..$^{12}$ As both listening and recording devices, both the electronic and digital stethoscopes are steeped in concerns of distorting an "original experience". Rather than see this as a hindrance to learning to listen, as a "misrepresentation", we argue that "learning" means not only being attentive and not distracted, but also incorporating, embracing, embodying distortion.

In this article we will build our argument specifically through bringing together our ethnographic and historical material on bedside and lecture teaching. The bedside events focus on the integration of the instrument into clinical bedside demonstrations. We will look here at methods of collective learning, with the electrical and digital stethoscopes being defined as either disruptive and/or innovative to pedagogical traditions and to learning auscultation. The lecture room events focus on the modalities and media involved in performing knowledge, on the different forms of simulation incorporated into learning.

9 Frederick and Dodge 1924.

10 Arlette Farge's text on the historian's material and bodily practices in the archive remains relevant, even in light of changes brought about by the digitization of archives and big data (Farge 1989). Related to this are Gabriella Giannachi's book (2016) on the archive and practices of archiving, and Lisa Gittelman's edited volume (2013) on the constructed nature of data and the different cultural practices (constitution, storage and interpretation) involved in establishing data sets across different disciplines.

11 Methods of media archaeology that bring different forms of historical remains together are also useful to this article's historical work on material culture and events: see Huhtamo and Parikka 2011.

12 Hirschauer 2006. 
In the final part of the article and in the conclusion we problematise the ethnographic recordings and historic renderings of the electronic and digital stethoscopes, underlining that the distortions inherent to such crafted mediatised events emerge even more strongly through cross-disciplinary dialogues. By considering the methodological practices of ethnography and history side by side, we argue for the centrality of distortion to such methods, showing how juxtaposing them highlights the importance of attending to our own recording practices as researchers, including their silences and gaps.

\section{Electronic and digital stethoscopes: A brief introductory history}

The stethoscope is an iconic medical instrument, one that may be described as the hallmark of a doctor. ${ }^{13}$ Historian of science and technology Jens Lachmund underlines that while listening had always been involved in medical diagnostic practices, it was only in the $19^{\text {th }}$ Century that listening (through a stethoscope) became an "epistemically priviledged and generalised routine", having a notable role in a new professionalization of medicine. ${ }^{14}$ Pointing out that the visual was "not the only form of sensory experience to undergo systematic elaboration and epistemic instrumentalization in modern science", Lachmund writes: "with the rise of auscultation [...] the auditory became viewed as a distinctive sphere of perception and functioned as the nexus of specific perceptual practices. Therefore, the physician's medical expertise at least partly became transposed into the realm of the auditory." ${ }^{15}$ In exploring mediate auscultation as one culturally specific example of an 'audile technique', communication studies scholar Jonathan Sterne has more broadly illustrated how epistemic programs of modernity articulated listening (a bodily technique) to reason, rationality, and science: audition became a site "though which modern power relations [could] be elaborated, managed, acted out". ${ }^{16}$

Notable shifts in the relationship of the doctor and patient emerge with the articulation of mediate auscultation and its particular listening dispositive. If immediate auscultation implied putting the physician's ear directly against the skin of the patient, French physician R.T.H. Laënnec's experiment of placing a cylindrical tube (1816), a monaural stethoscope, between one ear of the doctor and the patient's chest changed the nature of this close

13 Rice 2010.

14 Lachmund 1999, 420

15 Lachmund 1999, 428.

16 Sterne 2003a, 59. See also Sterne 2001; Sterne 2003b. 
physical proximity between bodies. ${ }^{17}$ The listening tool created an enclosed acoustic pathway to the patient's body, which would be more fully realised by the binaural stethoscope model (the ancestor to the common acoustic stethoscope) that appeared around $1850 .{ }^{18}$ The novelty of this binaural model was the plugging of both ears through two earpieces, which attached to a piece of rubber tubing and a chest piece (possessing a bell and a diaphragm). ${ }^{19}$ The rubber tubing permitted even more distance between doctor and patient, but the model made the sounds seem to come closer into the ear. ${ }^{20}$ In such a one to one, enclosed connection the doctor became isolated from others, perceiving phenomena otherwise unavailable to her/his own senses. This physical distance also created a mental one that permitted the patient's body to be objectified, turned into a dynamic field of action. ${ }^{21} \mathrm{Re}-$ ferring to Laënnec's method that was rooted in pathological anatomy, medical historian Jacalyn Duffin explains this field:

The mysterious changes inside the 'bony cage' of the patient's chest could now be brought to view - explored or examined - through the doctor's hearing. The stethoscope eliminated speculation about the range of possible diagnoses through 'signs' that indicated organic 'facts', independent of the physiological circumstances that had been their cause. ${ }^{22}$

Like Duffin, Jens Lachmund underlines the necessity of both intellect and sensory perception in this diagnostic practice, further explaining the history of auscultation as both "a process of perceptual standardization that [...] included the coproduction of an organized universe of analyzable auditory signs and an auditorally sensitive body that was able to decipher their meanings." ${ }^{23}$ Numerous scholars have also explored the invention of the stethoscope device and dispositive in hospital, as well as its cultural uses in contemporary hospitals and medical schools. Auscultation's space of knowledge has historically been constituted across sites of medical research and practice: the embodied, hands-on experiences happening in clinical medicine are of fundamental importance. ${ }^{24}$

17 See Laurent Tortil's beautiful photographic presentation of photos of Laënnec's stethoscopes housed at the Musée de la Médecine, Paris (2019).

18 Leared and Marsh binaural models appear in 1851; see Blaufox 2002, 39.

19 Groom 1964, 291.

20 Tom Rice has described this as "narrowcast" stethoscopic listening $(2012,310)$. It is a sensation of bringing sound inside the physician's ears: as if the "patient's body [is] penetrating the listener" $(2010,49)$.

21 The impact of changing diagnostic medical practices on the modern constitution of the patient body as medical object and the status of the patient's verbal account of disease - in which auscultation has a decisive historical role - have a special place in sociological studies of medicine; see Armstrong 1994, 19. On the specific ways in which the stethoscopic listening situation objectifies the patient, see Rice 2008.

22 Duffin 1998, 152.

23 Citation: Lachmund 1999, 439. Reference to Duffin 1998, 153.

24 In addition to Duffin 1998 and Lachmund 1999, see Tom Rice's ethnography (2013). 
To date, many of these histories of the stethoscope have neglected to focus on its electronic and digital forms, and how they are used in pedagogical practices.

Electronic and digital stethoscopes are technologies that fold throughout time. They are not conceived to replace the traditional binaural stethoscope, nor its practices. They actually have a relatively limited use in everyday medical practice, in comparison to the ordinary acoustic stethoscope. The electronic and digital stethoscopes have been designed to "enhance" diagnostic listening that would normally be done through the binaural acoustic stethoscope, reaching across the limitations of hearing difficulties and differences - which which include hearing disabilities, particularly challenging cases of diagnosis, and trying to find uniformity in relation to the isolated and subjective sensory perspective of the practice. ${ }^{25}$ In their description of the stethophone (1923), an early electrical stethoscope system made for teaching, Frederick and Dodge explain the applications:

With the development of vacuum tube amplifiers, the possibilities of reproducing and magnifying body sounds electrically were considered. It appeared that a device might be provided which would be useful not only in teaching but also in diagnosis, as an aid to physicians of subnormal hearing, in the reproduction of very faint fetal heart sounds or even in fields beyond the scope of ordinary stethoscope. ${ }^{26}$

As ethnographer and historian together, we wanted to explore these marginal instruments as a means of reflecting on processes of learning bodily practices, such as the physical aspects of listening to sounds in medicine.

Most simply, the electronic and digital stethoscopes introduce electricity, microphones, and even recording capacities into the auscultative experience. The electronic and digital stethoscopes' histories of use are entangled in multiple ways with the process of becoming skilled. They develop ideas of transmitting body sounds (mainly heart and lung sounds) across space, fixing them in time and augmenting their perception. They simulate the practice of individual listening through stethoscopic earpieces when auscultating, while permitting students to listen collectively to the professors' demonstration. These electrified experiments of auscultation depend greatly on amplification and transmission technologies and infrastructures. ${ }^{27}$ The development of the car-

25 The narrative that this electronic stethoscope is created first and foremost for students with hearing impediments is repeated time and again across sources. See, for example, "Electrical Stethoscope Aids" 1936, 12.

26 Frederick/Dodge 1924, 534.

27 The connection between medical schools and sites of technological research, such as Bell Labs or the Cambridge Instrument Company, are mentioned in medical research papers published between 1920 and 1950 on the subject of the application of electronic stethoscopes in medical training. In addition to an acoustics explanation on how the instruments augmented the sounds, such publications included detailed descriptions of the technical equipment requirements, the installation, the acoustic research behind the device, and ad- 
bon transmitter and microphonic contact devices permitted early $20^{\text {th }}$ century experiments of transmitting and recording body sounds. W. Eindhoven used such technologies to record normal heart sounds and murmurs in 1907.28 In 1910, S.G. Brown underlined connections to telephone research when describing one electric stethoscope prototype "that transmitted heart sounds over a commercial telephone line in London using a single microphone and electromagnetic relay." ${ }^{29}$ Squier amplified normal heart sounds using a carbon transmitter in 1921, but had concerns of 'microphone roar' interfering with the perception of body sounds when using carbon microphones. ${ }^{30}$

Electrical stethoscopes were first incorporated into medical practice as teaching aids largely due to the efforts of Drs. R.C. Cabot and J.C. Gamble and the installation of these devices into their classrooms at the Massachusetts General Hospital in the United States in June 1923. ${ }^{31}$ This device allowed up to 125 students to listen together. There was also a tea trolley model for collective bedside examination demonstrations in the hospital ward. We will explore both variations of this 1923 system later on in the article. Over time, the device became more compact, resulting in a portable model for individual consultations such as the 3-A portable electrical stethoscope (shown in fig. 1):

The instrument is completely contained in a fabrikoid bound case, smaller than a doctor's instrument bag. It measures 12.5 inches in length, 8.75 inches high, 4.75 inches deep. It consists essentially of a sensitive microphone or transmitter of special design to pick up heart sounds, a vacuum tube amplifier to amplify them and a high quality receiver, which reproduces them. The receiver is so arranged that the physician may attach it to the tubes of his regular stethoscope $[\ldots]^{32}$

By the 1950s the quality of sound rendition had improved, and additional capacities to be able to auscultate more obscure lung sounds and to record sounds became feasible. ${ }^{33}$ What is called the digital stethoscope still carries the nomenclature of this 1950s electronic model (such as the Littman 3M electronic stethoscope). It is a sophisticated wireless version, which reflects processes of technological miniaturization. ${ }^{34}$ In this article, we are juxtaposing ethnographic and historic events involving these instruments at their earliest and at their most recent stages of development.

vice on device settings. See Frederick/Dodge 1924; Butterworth/Poindexter 1948; Kinnier Wilson/Fothergill/Taylor 1956; Rappaport/Sprague 1941.

28 Eindhoven 1907; Frederick/Dodge 1924, 535.

29 Brown 1910; Frederick/Dodge 1924, 535.

30 Ibid, 1924: 535.

31 See Cabot 1923; Frederick/Dodge 1924. 535.

32 "Electrical Stethoscope Aids" 1936, 12, 19.

33 See Rappaport/Sprague 1941; Butterworth/Poindexter 1948.

34 For more information and references on the evolution of the digital stethoscope, see Swarup/ Markaryus 2018. 
Fig 1. The 3-A portable electrical stethoscope, in: "Electrical Stethoscope Aids Heart Diagnoses" Pickups Journal of Western Electric, May 1936.

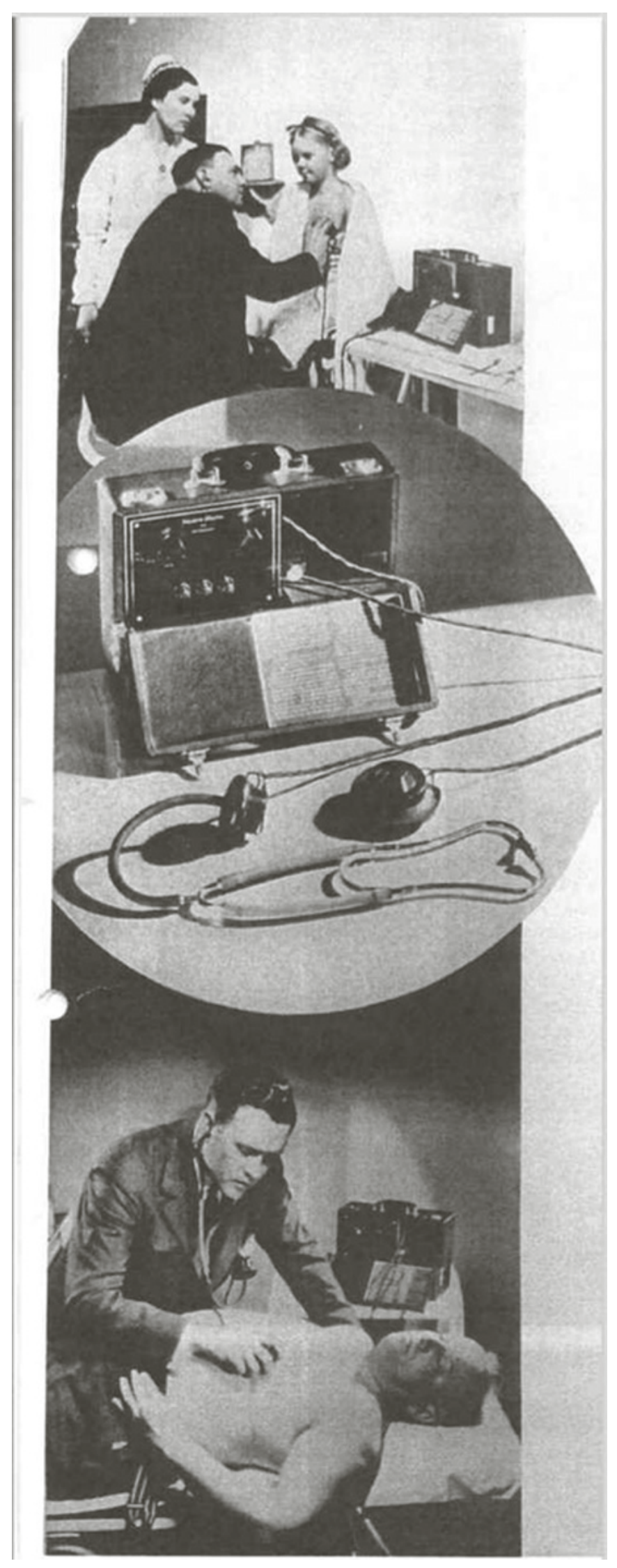


We turn now to look at how both the electronic and digital versions are used in teaching medical students, historically and ethnographically, beginning at the traditional site of the bedside.

\section{The Bedside}

From Anna's fieldnotes:

I am standing amongst a group of six medical students, around a patient's bed on the respiratory ward of the teaching hospital where I am doing fieldwork. I am observing one of Professor Smith's (pseudonym) ward rounds. Professor Smith leans attentively towards the patient, named Mary (also pseudonym) who was admitted the night prior due to shortness of breath. Professor Smith asks if she minds if one of the student doctors practices an examination with her. Mary nods.

The student performs her clinical examination and reports the findings back to the Professor and the group. Then it is time for the other students to have a listen to Mary's chest, for it turns out that she has "an interesting sign". Each student listens with their stethoscope. One student shakes their head; she can't hear the sign. A few others nod and repeat the diagnosis they have heard the Professor mention moments before. One of the students steps up to the bed with a stethoscope, which looks slightly different from the others, and I realise that this is a digital stethoscope. She listens using a technique like the others, her fingers holding the end piece slightly differently. She also listens for longer than the others, too. The Professor steps forward slightly and says to her, with a little grin, "perhaps you need to turn the volume up".

Professor Smith's comment is made cheekily, although has deeper meaning. If we look at his own tools we see that he has a cheap red plastic stethoscope, one he either grabbed from the wards or more likely, chose deliberately for its cheapness. For Professor Smith, the skill of listening to sounds is not in the instrument. He doesn't believe in digital stethoscopes, he finds them distracting.

From Melissa's archives notes:

A black and white photograph (fig. 2). One that has the grainy quality of being reproduced in a magazine, its contours having faded with age. A female patient is lying in a hospital bed just off centre. Her face is sombre, white, mask-like: her lips are pressed, her gaze distracted, her hair neatly and symmetrically plaited. The colour of her skin matches that of her bed gown. Her body disappears in the large rectangular bed, except for her chest and one 


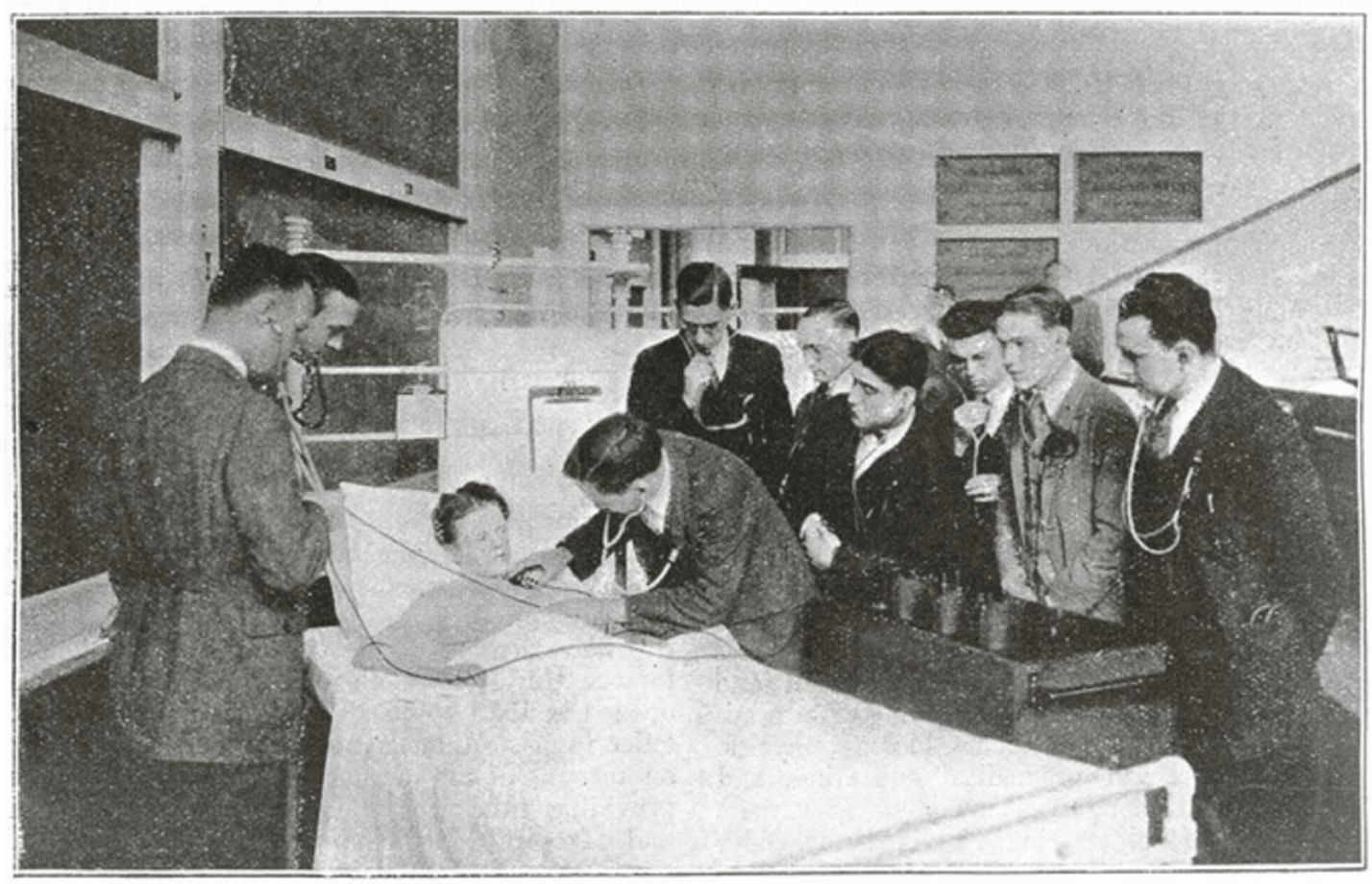

A Bedside Demonstration.

[General Pholographic Agency.

Fig. 2. A bedside demonstration of the collective stethoscope. Hospital and Health Review, September 1924 (Credit: Wellcome Collection. CC BY).

hand that rests heavily on top of the draped, white bed sheets. It's a sharp contrast to the machine made of dark wood and metal, the size of a tea wagon that has been rolled up next to the patient's bed. It has a control panel with batteries and jacks and knobs. A man in a dark brown suit, the professor, is standing between the machine and the female patient. He wears a white stethoscope in his ears and he bends over the patient in a gesture of auscultation. One hand rests on the patient's abdomen, his forearm grazes her hand. His other hand holds a round diaphragm placed on the patient's chest, over her heart. Something is amiss though, for nothing connects the black stethoscope diaphragm to the white earpieces; no tubing makes an acoustic pathway between patient and doctor, and the stethoscope has neither a small bell, nor the tubing of a regular stethoscope. Look again. The white tubing has been attached to the professor's dark suit lapel. In the absence of the familiar small bell, the professor's hand is spread wide around the much larger microphone-diaphragm. The posture of the professor seems freer: there is more space. A thin black wire actually runs out from the diaphragm on the patient's chest down her side towards the machine.

A group of eight clean-shaven, well-coiffed young men, the students, stand on either side of the bed. Dressed in dark black and brown suits, they are 
carefully positioned to frame, not block, the view of this professor, the female patient, and the machine. Like the professor, white stethoscope earpieces are fixed in their ears, but no dangling tubes and diaphragms of this familiar acoustic model are visible. Bits of the tubes round up and are pinned neatly to each of their coats.

Six students stand to right of the bed, fanning out behind the machine. Two others stand to the left of the bed, with their backs slightly turned. These turned backs use the theatrical effect of the 'fourth wall' to create a feeling of realism. Further, the students strike different poses that demonstrate they are listening attentively. Some have fixed their gazes on the woman patient. Look more closely at the student leaning on the machine who watches the professor's hand and the position of the diaphragm on the chest. Three others have dropped their heads or diverted their gazes from the doctor and patient.

Even if the eyes are not all directed to the same place, these gestures of leaning and being concentrated tell us that the men are sharing an experience. The ways in which one can be present with the stethoscope have multi-

plied. The wires are material traces that suggest a controlled space, reuniting all those individual gestures back to the professor who is controlling what they are hearing through the machine. Just as one wire moves down the patient's body to connect into the machine, another thin black line moves across the white space of the bed, connecting students' bodies. Wires make listening simultaneously and collectively possible, while maintaining the felt experience of listening through earpieces.

\section{Bedside events: individual and institutional definitions of collective listening}

The ethnographic event describes an established training ritual, practiced in hospitals since the $19^{\text {th }}$ Century, when it was considered important not to learn about the body on cadavers but on real patients. Since the time when hospitals became a place for learning alongside healing, the two processes have become intricately intertwined. In this context, where the acoustic binaural stethoscope is a fixture, the student's use of the digital stethoscope stands out as anomaly. Its disruption occurs through its different physical appearance, through Professor Smith's comments on being able to "turn up the volume", to its capacity to "enhance" and record body sounds. The photo "A Bedside Demonstration' (1924) on the other hand represents an initiative of the pedagogical institution to standardise the learning experience through creating a controlled, shared, singular experience of a body sound. This tea- 
wagon consulting model for wards was developed as part of the large-scale stethophone system conceived for the lecture hall (which we will speak about below) and precedes the portable individual electrical stethoscope (such as that mentioned above in Fig. 1). According to Frederick and Dodge, this model includes an electromagnetic transmitter, a three-stage amplifier with a potentiometer control, a selected group of electric filters, a multiplicity of output receivers for observers:

The whole is assembled in a substantial cabinet on wheels resembling a "tea-wagon". It requires for its operation a six-volt battery and a 130-volt "B" battery. These are housed in the lower part of the cabinet. Ten jack positions are provided to permit this number of persons to listen simultaneously around the stethophone. ${ }^{35}$

The bedside event is, from both ethnographic and historical perspectives, about the introduction of a new technology within an established practice, which as much frames the performance of a routine skill, as it does define its own particular modes of use and where it fits into learning embodied knowledge. Professor Smith finds the digital stethoscope a distraction in the learning of listening skills, and perhaps suggests that the act of being able to manipulate the body sounds one hears, with volume buttons, is incompatible with the listening skills and sensory memory required for good doctoring. The enhancement of the instrument distorts in that it alters what is heard through a "classic" sensory technique.

The ethnographic vignette and the historical photograph are connected through the idea of collective listening acts, albeit very differently. In the ethnographic vignette it is the student who owns a digital stethoscope and uses it for individual reasons to help construct her practice. Emphasis on memory is key, and acts of playback and sharing recorded sounds occur outside the hospital ward. One does not simply listen to sounds, the sounds evoke the experience of the particular learning event. This highlights the incorporation of the marginalized technological gadget into personal experience.

The photo is very much the opposite as it is directed at the group listening through the same apparatus. Collective listening devices in medical teaching go back to the 1880s. For example, the Paul teaching stethoscope model became available in 1887. The Bowles multiple stethoscope, developed in the early $20^{\text {th }}$ Century, consisted of multiple rubber tubes and earpieces connected to a single diaphragm of a binaural stethoscope that allowed six to twelve students to listen to a single patient at once. Yet such collective stethoscopes had inconveniences: not only were students huddled together around the patient, but the extra rubber tubes made it difficult to perceive the sounds 
correctly. ${ }^{36}$ While this electrical bedside model developed in 1923 seems to make listening to the same sound by a group easier, the wires once again changed the dynamics of proximity that were discussed above. This cumbersome tea trolley model created new kinds of contact and dispositions between the bodies of the patient, the doctor, and the novices. Focus is placed on the connection to the instrument: in the image the students lean in slightly to illustrate their participation in the actual event, even as they are looking away from it, often gazing down as if to focus on something interior. Some hold their earpieces more tightly into the ear canal. These gestures are easily recognizable components of the iconography of modern mediatized listening, more particularly of the act of 'listening through' in which the connection to the instrument is key to hear and concentrate on acousmatic sounds. ${ }^{37}$

In medical training, collective listening through the digital stethoscope is similar to listening for example through stethoscopes with multiple earpieces. This correlates with the idea of sharing experience and attempting to eliminate subjectivity in other collective sensing practices in medicine, such as collective viewing down microscopes..$^{38}$ Once again in our cases of the historical and ethnographic material juxtaposed, we see collective listening very differently. In the ethnographic vignette it's the student that owns a digital stethoscope and uses it for individual reasons to help construct her practice, whereas in the photo the students are all hooked up, sharing the listening simultaneously. Let us move now to a much larger teaching event, the lecture, where collective listening acts are also enacted through the digital and electronic stethoscope.

\section{The Lecture}

From Anna's fieldnotes:

An eminent cardiologist in my field site hospital is giving one of his famous heart sounds lectures. When I arrive the lecture hall is dark as the lights have not been turned on yet, although soon I see a hospital bed, centre stage,

36 See the striking photo of twelve students using the Bowles stethoscope, in Cabot 1930, 146 147; see also Blaufox 2002, 63-65.

37 For more on the iconography of late $19^{\text {th }}$ century listening dispositions, see Sterne 2003; Van Drie 2015.

38 The idea of the collective stethoscope is similar to Nachet's triple-microscope that enabled three observers to view the object simultaneously through a series of reflectors and tubes. See the description of Dionysius Lardner (1854, 85, entry 89). 
around which the chairs fan out. Next to the hospital bed is a lectern and above the bed a large canvas for projecting PowerPoint slides.

Soon the lecture theatre fills up with second year medical students, and the cardiologist strides in. He introduces the students to the patient, John (pseudonym). There are questions posed regularly to the students, slides with pictures of fluid dynamics and symptoms of cardiac disease. The cardiologist also plays $\mathrm{mp} 3$ recordings of heart murmurs. There is quiet in the room, and he turns up the sound so that the booms and beats fill the lecture hall. These sounds do not stand alone, of course. They are played alongside anatomical pictures and graphical representations of heart murmurs on the PowerPoint slides (see fig. 3). A student puts their hand up. Can the lecturer do his aortic regurgitation imitation? The Professor is chuffed and starts moving his mouth and gesticulating in imitation of the pathology. The students are delighted.

Halfway through the lecture the cardiologist invites the students to come to the front of the lecture hall and examine the patient. Eventually the whole class proceeds to come on stage, each listening with their multi-coloured

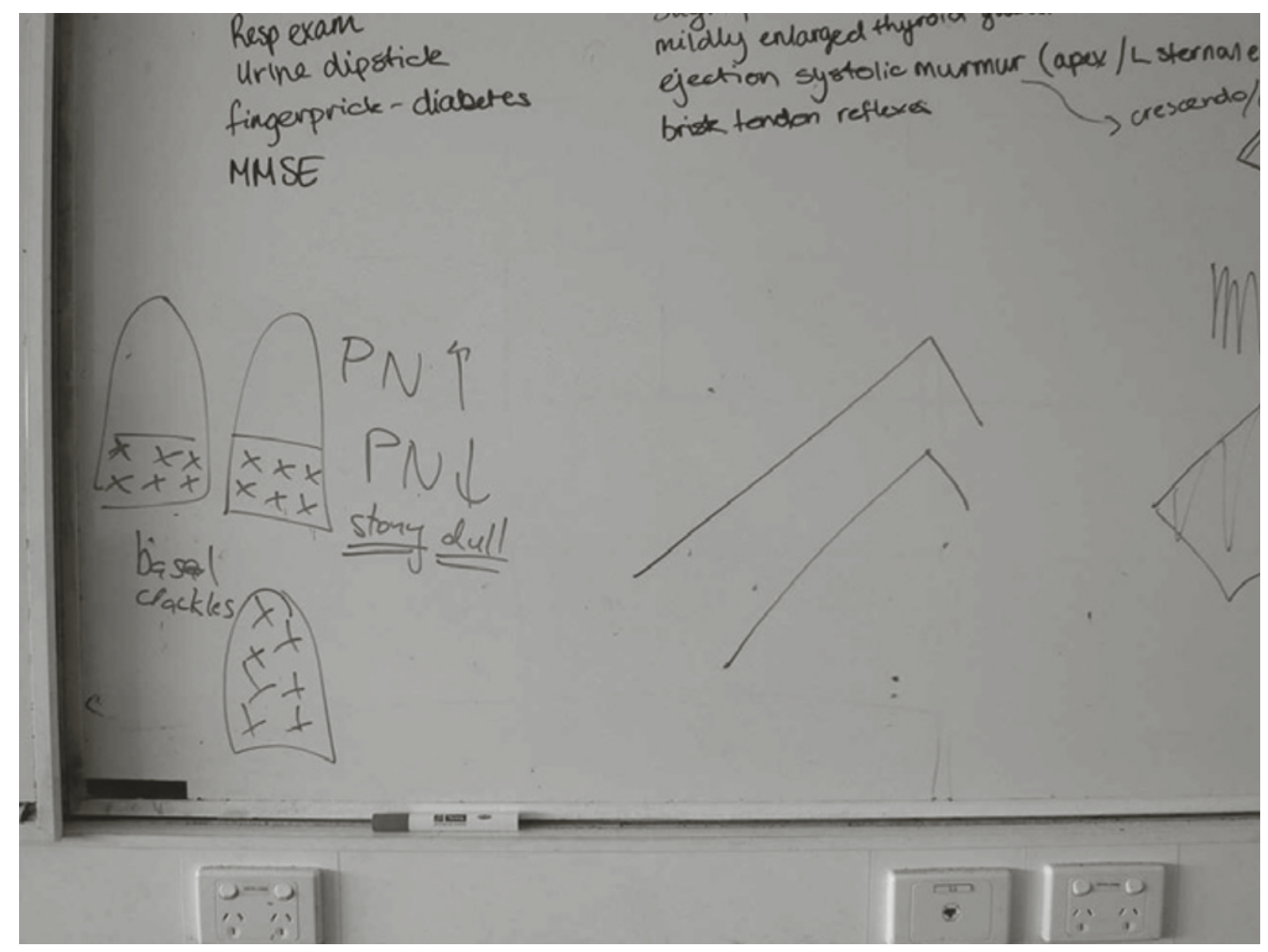

Fig. 3. Heart and lung sounds on a whiteboard, Melbourne, Australia (photo credit: Anna Harris 2013). 
stethoscopes. John lies back patiently on the hospital bed. The cardiologist gesticulates to his audience and declares that this man's heart sounds are better than any recording he could find on the internet. The students say that they should record the sound, one of their group has a digital stethoscope! Is it OK with him, the student asks gently? John nods and she records his sounds, sounds, which she will send to the lecturer for him to use in another lecture in the future.

From Melissa's archive notes:

Meandering through ... a pair of simple, black and white schematic diagrams, $\mathrm{n}^{\circ} 9$ and $\mathrm{n}^{\circ} 10$, portray an early application of telephone technology to medical pedagogical practice (fig. 4). Diagram n ${ }^{\circ} 9$ depicts the "wiring installation in an amphitheatre for teaching purposes" and has three components (group of three drawings on the left). The first shows the bird's eye view of the amphitheatre with its stage and seating arrangement. The stage is depicted as a white space containing a rectangle labelled "stethophone". The auditorium fans around with the seats represented by rows of open circles
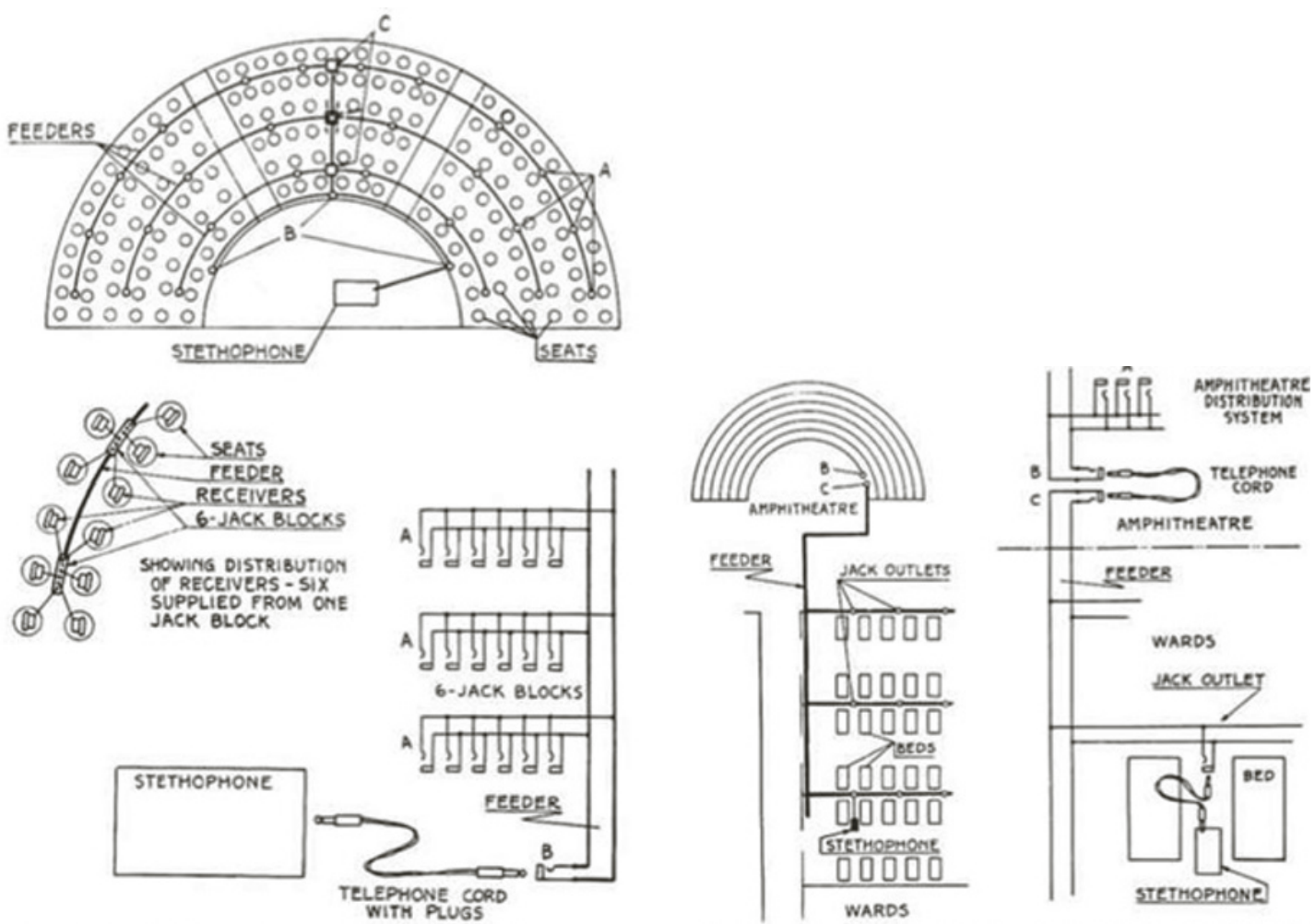

Fig. 4. The Stethophone, 1923, Schematic Diagrams n ${ }^{\circ} 9$ (on left) \& 10 (on right), in: Frederick/Dodge, 1924, 544-545. 
(top left). The second drawing (bottom left) illustrates how the telephone circuit is created through feeders extending out through the auditorium. Three ' $\mathrm{B}$ ' feeder points located along the stage ramp are connected by telephone plug to the Stethophone on stage and to the clusters of jack blocks 'A' in the auditorium. Finally, the drawing (middle left) of ${ }^{\circ} 9$ is a close-up of the jack blocks which contain sets of six telephone receivers each, one receiver per seat, three in front and three in back of a series of seats. Some rows plug stethoscope earphones in front of them, others plug in in back.

The technical description accompanying this diagram of the Stethophone resembles that of a telephone (indeed, it is published in the Bell System Technical Journal): the system was equipped with electromagnetic transmitters, a "special form of mouthpiece for picking up body sounds, a threestage vacuum tube amplifier and a distribution system to accommodate as many as 125 students with single head receivers on which individual ordinary stethoscopes were held." ${ }^{39}$ Amplified body sounds of a patient located onstage would be listened to either through attaching the open bell of an acoustic stethoscope to the receiver, or by using specific rubber tubing and earpieces of a stethophone system that greatly resembles an acoustic stethoscope. ${ }^{40}$

The second drawing of $\mathrm{n}^{\circ} 10$ (fig. 4 , to the right) shows how that amphitheatre distribution system is connected to the stethophone in the ward through wires and telephone jacks. This could bring the sounds of particular 'rare' cases into the lecture: "It is often desirable to reproduce in the lecture hall, the heart and chest sounds of patients too ill to be moved". ${ }^{41}$ This system also impacted the performance practice of the professor and a change in the relation to the patient, for one could not only listen to the inside of the patient's body, but talk through it:

In classroom lectures, the instructor can make announcements to the students and point out features of particular interest in a convenient and somewhat novel manner without requiring the removal of the stethoscope from their ears. The human body acts as a sounding board for sounds in the air - that is, when words are spoken in the vicinity of a patient, the flesh and bone structure vibrates to these sounds. This is particularly true of the areas commonly used in auscultation. The transmitter, resting on the flesh, will pick up these vibrations together with those originating in the body of the patient. The instructor may, therefore, talk to his students by directing his words at that portion of the body to which the transmitter is applied ...42

39 Frederick/Dodge 1924: 536.

40 Ibid. 542.

41 Ibid. 542.

42 Ibid. 545, our emphasis. 


\section{Lecture events: oscillations between live and transduced experience}

In both these ethnographic and historical reconstructions, event is performance, whereby a multitude of factors contributing to the staging of a skill are foregrounded. They highlight how scenic demonstrations contribute to the production and performativity of knowledge. In these two events sounds are performed in many ways: through bodily acts of mimicry, through speaking through a wired body, through icons on a PowerPoint, through listening while watching an empty stage. In the ethnographic vignette, the student's digital stethoscope creates new sound recordings that will be integrated and "re-enacted" in a future pedagogic event, as well as being piece of personal documentation for an individual student. Acts of archiving sound become possible in the ethnographic lecture. With the stethophone (1924) we witness the use of the technology to continue to acclimatise students to the binaural, enclosed listening experience of the stethoscope, which was simulated in the lecture amphitheatre. Bodily memory is at play here through the continued use of the stethoscope earphones, even when physically removed from the patient. ${ }^{43}$

The spatial arrangement of the lecture, its scenic "dispositif", is equally important to questions on how the lecture performance constructs learning: performers (notably the professor) and the audience are placed in a frontal configuration, but there are all kinds of interactions occurring, which are necessary for the lecture event to occur. If the ethnographic vignette underlines certain dialogues and participation occurring between the professor, volunteer patient and the stage, the stethophone with its mediatisation of the auditorium seems to create a number of separations. If listening collectively to the same sound should in theory create uniformity and a literal "shared listening" experience (once again, everyone is hearing the same sound at the same time and being guided by the professor), there is also a multiplication of different sensory scenarios through these possibilities to electrically record and transmit sound.

For example, within the auditorium, there is a contrast between watching the movements of the professor onstage and the transmitted voice pumped through earphones: this technological synching creates a sort of audio-visual distortion. In this first description of the stethophone lecture hall (fig. 4, $\mathrm{n}^{\circ}$ ), one still sees the sound source. In the second description of the lecture hall listening through the stethophone into the ward (fig. 4, $\left.\mathrm{n}^{\circ} 10\right)$, one loses it.

43 See Rappaport/Sprague 1941. The decision to not use a loudspeaker is also related to acoustic distortion. See the article on loudspeaker teaching systems in Phillips 1949. 
In this latter case, the experience becomes mainly an acousmatic or radiophonic one: the principle focus is on transmitted sounds; and we can imagine the audience looking at the seat in front of them, closing their eyes, looking at other audience members or looking at an empty stage..$^{44}$ Indeed, it isn't surprising that the idea of creating a virtual consultation practice was imagined as an early application of these devices..$^{45}$

From this consideration of the scenic design, the intermedial performance recounted in the ethnographic vignette through a very specific arrangement of different modes and dispositions of listening - recorded and live sounds, imitations, listening over live speakers, listening through the stethoscope, watching listening, listening and looking at images - finds resonance in the different combinations and variations of sensory modalities (live and mediatized) constructed through the stethophone's telephonic apparatus.

In both cases there is the sense of "an original" situation of experience, which becomes distorted in the effort to share sound. The lecturer says the mp3s of lung sounds are not as good as the patient's. But what does this mean exactly? Certainly, the digital recording technology is able to record the lung sound or heart beat with precision, and probably more distinctly than a human ear listening through the stethoscopic earpieces. No, the question of original here is not mainly about the quality of the recorded sound, nor even of introducing this additional layer of sonic mediatisation into the auscultative moment. The question of an "original" event relates to changes in the listening apparatus. The definition of an expert ear is dependent on a particular wielding of the acoustic tool and a particular multi-sensorial understanding of lung and heart sounds. This sense of an original sensory experience emerges throughout the vignettes above within accounts of "original" ways of listening, "traditional techniques", acoustic stethoscopes and allusion to various ways of accessing the patients' bodily sounds. It's the change to the acoustic way of listening (changes in the bodily postures, the sights and smells of the patient's body, the skin to skin contact) that is at stake here when these new electrical techniques are included to try to insure that all students hear in the same way, or hear difficult sounds more easily through a certain acoustic filtration. Details and entanglements do matter in creating 'auditorally sensory bodies'.

44 See Lacey 2013 on the politics and experiences of mediatized listening.

45 "The stethophone or radiostethophone has now made it possible for doctors on shore or on board a large liner to listen to the heartbeats of a sick man on some smaller boat that has no medical officer. Diagnosis can then be given and treatment prescribed by wireless," in "Heartbeats by Radio" 1924, 272. See also Winters 1921. 
One of the most radiophonically poetic distortions is when the stethophone upsets interactions with the patient. Situated in the ward, the professor was invited to speak to students in the lecture hall not only through the microphone positioned on the chest of the patient, but to speak through the patient's body, to use its resonant potential to transmit commentary. This certainly disrupts traditional presentation styles: to listen and then explain what is heard. More importantly, it is a striking act of patient objectification. The wires are now fused with the patient's body: bone, cavity and fluids have become part of the medium of transmission.

\section{Juxtaposition and distortion}

One of the marketing descriptions common to both electronic and digital stethoscopes is that they are able to record sounds "more accurately", that they offer a representation (whether amplified transmission or recording) of the original that is clearer, measurable, repeatable, comparable and shareable as an "objective" truth, as opposed to the more ephemeral, personal and subjective experience of listening with an acoustic stethoscope. The development of the electronic stethoscope was connected to experiments of mechanical objectivity, notably truth-to-nature pursuits of graphic methods, which developed recording devices to record the rhythms of different physiological functions. ${ }^{46}$ These experiments attempted to visually fix movement and to universalise it. Our reflection on these stethoscope devices in practice points to all sorts of different ways that the registering and transmission machines pose problem to such objectivity.

In this paper we have problematized that the recordings made by these stethoscopes, including that the sensorial experience of the technologies, are "original". Instead, we have tried to distance ourselves from notions of accuracy associated with sound reproduction technologies (the "copy of original') by situating the recording within the broader performative event, showing how it means within a specific historical context. We defined each listening event as an intra-active set of practices enacted through and representing a choreographed arrangement of people and things, places and sounds, bodies and wires.

46 For example, Carl Ludwig's Kymograph (1850) was a self-registering device that measured and visualised the movements of blood-pressure. On the inclusion of $19^{\text {th }}$ century sound registering devices in medicine, see Volmar 2013. On mechanical objectivity see Daston/Galison 2007. 
The ethnographic vignettes reconstituted from notes and memory, are not presented here as accurate representations of an original lived experience, but rather, an event that arises in the writing. What we mean explicitly by this is that ethnography is not an objective recording of observed data, but rather that the ethnographic narrative is constructed in the very act of storytelling and writing. This correlates with historiographical approaches of material culture, which considers that acts of performing with artefacts and (re)enacting past practices or actions are powerful tools for engaging different and multiple dimensions in our framing of historical events. We want to reflect on this further now, drawing specific inspiration from Stefan Hirschauer's (2006) text on ethnographic description and Patrick Feaster's explanation of phonography and the impossibility of duplicating sonic phenomena (2015).

Hirschauer teases apart the nature of ethnography, specifically documenting practices, and argues that "ethnographic writing puts something into words that, prior to this writing, did not exist in language". ${ }^{47}$ As we described above, there is no original, the event arises in the writing, in the teaching, in the learning. The ethnographer outdoes, "what the participants could have known about the situation". ${ }^{8}$ Just like the electronic and digital stethoscope, the technical preservation (through writing or archiving material) does not reside in the ability to make an exact copy but in the creating of something entirely new, as Hirschauer writes: "the social original is a sociological fiction, an idealization intrinsic to recording technologies". ${ }^{49}$ There is no original digital stethoscopic listening event but rather an ethnographic retelling.

The work of historian and (sound) media archaeologist Patrick Feaster on the impossibility of making a sound recording copy complements Hischauer. Reflecting on the multi-faceted history of phonography (a sound-writing machine), Feaster writes the following on the topic of an original sound and its copy:

[...] even authentic copies of real sounds are copies, so the same paradigm also invites judgments about which characteristics of originals can be satisfactorily preserved in "reproductions" and which cannot. Scholars have usefully examined the construction of phonographic "fidelity" as a measure of audible likeness between original and copy [...] But even perfect audio fidelity wouldn't address the intrinsic and total loss of aura posited by Walter Benjamin's theory of mechanical reproduction (1969). I've put forward the additional concept of performative fidelity to recognize the degree to which the social force associated with a sound is accepted as carrying over into its reproduction: for example, the question of whether phonographic music can substitute functionally for "live" music $[\ldots]^{50}$

47 Hirschauer 2006, 414.

48 Ibid. 420, our emphasis.

49 Ibid. 421.

50 Feaster 2015, 144. 
After considering how belief has a role to play in how sound copies have come to functionally substitute originals, Feaster returns again to the impossibility of making a true sound copy. He explains this first through acoustics: when recording a sound on a phonograph, for example, an aerial sound that has a three-dimensional structure is transformed, or "rephenomenalised", into two-dimensions, just like a pair of human ears. ${ }^{51}$ So the sound copy is never materially the same thing due to the process of translation that happens through the machine and which resembles in this case the mechanism of the tympanic membrane. This reflects the inherent distortion present in auscultation, as one listens through a stethoscope. Second, Feaster turns to the question of socio-material contexts and the nuances of sound recording practices. He points to the oversimplification in "the equation of phonography with the recording and reproduction of sound [which] also omits the creative elements of phonographic practice and culture." Here Feaster points to the situatedness of recordings, as we did above. In thinking about recordings as sonic events, which are complex and mutable entanglements of matter and medium, Feaster shows that engaging different dimensions in our historical framings or creation of events is important and possible. He first draws on technical and acoustic knowledge to consider acoustic distortion. Secondly, he references practices of representation, drawing on media, theatre and performance studies.

Such ways of doing history may consider the roles that non-verbal, often undocumented interactions (between humans and non-humans), physical conditions, and gestures have in knowledge production. Methods of historical work based in the senses and in movement, self-reflexively framed through performance, can relate the depth of historical events. These methods include practices of re-enactment, reconstitution, and re-mediation, which span across performance studies, art history, history of science and techniques, music and media studies. ${ }^{52}$ Returning to the historical events of this paper, certain statements about acoustic distortion were cited from

51 Ibid. 145.

52 We cite a few of the epistemic sites and collaborations in which Melissa has been able to explore such performative and bodily approaches: 1 . Arts based research and phonograph recording demonstrations with artist and sound media archeologist Aleks Kolkowski (at the Technical U., Berlin 2015; Maison Française d'Oxford, Oxford 2019), see Van Drie 2019; 2. Considerations of intersections of the history of artistic and scientific performances in the Performing and Knowing workshop and reading group organised by Charlotte Bigg, Rafael Mandressi, Marie Thebaud-Sorgier at the Maison Française d'Oxford and the Centre Alexandre Koyré (CNRS, Paris), 20 April 2018; 3. Theoretical reflection based on reconstruction experiments at the "Histories and Practices of Multi-Channel Sound Reproduction Workshop", organised by Andreas Fickers \& Stefan Krebs, Luxembourg Centre for Contemporary and Digital History (C $\left.{ }^{2} \mathrm{DH}\right)$, February 2-3, 2017. 
technical journals. Additionally, Melissa's reading of the performative distortions of the stethophone's learning apparatus were informed in more creative ways, by going into archives, museums and collections, and encountering different sound devices. These included listening through old acoustic stethoscopes and $19^{\text {th }}$ century telephone earphones; re-enacting old phonograph demonstrations by singing into a phonograph to record a wax cylinder; simulating learning experiences by following the instructions of training cassette tapes teaching lung sound auscultation..$^{53}$

Returning to ethnographic events, they are descriptions of Anna's time in the field where she spent time learning skills with medical students, speaking to teachers, interviewing stethoscope sales people, holding focus groups with medical students and perusing medical bookstores. She also experimented with the digital stethoscope herself and took notes autoethnographically. The students shared sounds that they had recorded on their digital stethoscopes with her and she took a digital stethoscope home for a week and experimented again on her own body, recording the sounds.

The descriptions of these ethnographic events are artefacts. Our methods of recording, have not conserved a social process, but rather, as Hirschauer points out, "de- and recontextualise[d] them in a highly complex fashion". ${ }^{54}$ It is important for us to reflect on this not only for methodological reasons, but also because this offers insights into the events that we have decontextualised - events which are themselves concerned with isolating body sounds for learning, in the process de- and recontextualising them in extraordinary ways. The doctors attempt to isolate and attend to the sounds of this body and want to avoid at all costs the nuisances of distortion. Yet distortion is key to the event, to the learning and the sounds being heard. Our methods are similar. As Hirschauer writes, "if every description is an addition, omission, accentuation, and presentation, and if all these things are considered 'distortion' according to the truth criteria of the recording, then it has to be said that many ethnographies suffer from a lack of distortion." ${ }^{55}$

For us, juxtaposition of ethnography and history helps to highlight the distortions of which Hirschauer speaks. While anthropologists may draw on the histories of the places they study, historians increasingly use sensory ethnographic techniques that explore deep description, emplacement and processes. Methodological links between anthropology and history are not always made and we think such links are productive. By looking at ethnographic

53 On Melissa's phonograph reconstructions, see Van Drie 2019; on medical teaching cassette tapes, see Van Drie 2013.

54 Hirschauer 2006, 422.

55 Ibid., 438. 
events alongside historical ones, the constructed nature of their descriptions is highlighted, and the distortions that each author used to craft the text to make an argument was brought closer to the fore. In doing so, we problematise a neat retelling of "being with" participants and the privileged access that anthropology often claims to have to skilled practice.

\section{A brief conclusion}

The electronic and digital stethoscopes are both listening and recording devices, which are part of highly performative multisensory learning events in medical education, in which bodies, wires, skin, metal, patients, sounds, screens, beds and rooms intra-act. These events as we construct them, are revealing in the ways in which they ask learners to attend closely, to attempt to isolate their senses and avoid distraction. The digital and electronic stethoscopes help to do this by amplifying sound, extending the listening event beyond the thresholds of hearing, beyond the classroom into the hospital, blurring delineations of proximity and distance. At the same time, they also disrupt the learning moment, leading to new gestures or interrupting traditional teaching routines. All of the staged performances are steeped in distortion.

Learners not only need to learn to attend and avoid distraction, but also how to deal with this distortion. The need to attend to distortion simultaneously highlights the fallacy of "the original" which one learns about, and the fallacy of a universal body or set of body sounds. Just like attention and distraction, distortion cannot be separated from technologies of learning. The stethoscopes we studied highlight the importance of this and how distortion is incorporated into sensory training. The more they try to isolate the listener from other sounds, the more distortion occurs. As researchers we also need to attend to distortions, rather than considering our archival or ethnographic retellings as recording practices. It is through distortions that researchers make arguments and give words to the silent. 


\section{Bibliography}

Armstrong, David, "Bodies of Knowledge/Knowledge of Bodies", in: Colin Jones/ Roy Porter (eds) Reassessing Foucault: power, medicine and the body (London/ New York 1994) 17-27

Atkinson, Paul, Medical Talk and Medical Work: The Liturgy of the Clinic (London 1995)

Barad, Karen, Meeting the Universe Halfway: Quantum Physics and the Entanglement of Matter and Meaning (Durham 2007)

Blaufox, Donald M., An ear to the chest: an illustrated history of the evolution of the stethoscope (Boca Raton 2002)

Brown, S.G., "A telephone Relay," Journal I.E.E. May 5 (1910)

Butterworth, Scott J./Charles Poindexter, "A multiple channel electronic stethoscope for teaching and demonstration", American Heart Journal 36 (1948) 112-114

Cabot, Richard Clarke, "A multiple electrical stethoscope for teaching purposes", Journal of the American Medical Association 81 (1923) 298

Cabot, Richard Clarke, Physical Diagnosis, $10^{\text {th }}$ edition (New York 1930)

Daston, Lorraine/Peter Galison, Objectivity (Cambridge, Mass. 2007)

Duffin, Jacalyn, To See With a Better Eye: A Life of R.T.H. Laennec (Princeton 1998)

Eindhoven, Willem, "Die Registrierung der menschlichen Herztöne mittels des Saitengalvanometeers", Archiv für die gesamte Physiologie 117 (1907) 461

"Electrical Stethoscope Aids Heart Diagnoses", Pickups Journal of Western Electric (May 1936) 12-19

Farge, Arlette, Le Goût de l'archive (Paris 1989)

Feaster, Patrick, "Phonography", in: David Novak/Matt Sakakeeny (eds.), Keywords in sound (Durham 2015) 139-150

Frederick, H.A./H.F. Dodge, "The Stethophone. An Electrical Stethoscope", The Bell System Technical Journal 3 (1924) 531-549

Giannachi, Gabriella, Archive everything: mapping the everyday (Cambridge, Mass. 2016)

Gittelman, Lisa. (ed.), Raw Data is an Oxymoron (Cambridge, Mass. 2013)

Groom, D., "What Constitutes an Efficient Stethoscope", in: Bernard L. Segal (ed.), The Theory and Practice of Auscultation: The Ninth Hahnemann Symposium (Philadelphia 1964) 291-299

Harris, Anna, "Listening-touch, affect and the crafting of medical bodies through percussion", Body \& Society 22 (2016) 31-61

Harris, Anna/Melissa Van Drie, "Sharing sound: Teaching, learning and researching sonic skills", Sound Studies: An Interdisciplinary Journal 1 (2015) 98-117

"Heartbeats by Radio. A new use for wireless", The Hospital and Health Review, September (1924) 272-273, URL: https://wellcomecollection.org/works/nh76vb4w

Hirschauer, Stefan, "Putting things into words. Ethnographic description and the silence of the social", Human Studies 29 (2006) 413-441

Huhtamo, Erkki/Jussi Parikka, Media Archaeology Approaches, Applications, and Implications (Berkeley 2011)

Ingold, Tim, The Perception of the Environment: Essays on Livelihood, Dwelling and Skill (London 2000) 
Johnson, Ericka, "Surgical simulators and simulated surgeons: Reconstituting medical practice and practitioners in simulations", Social Studies of Science 37 (2007) 585-608

Kinnier Wilson, A. B./Leopold Fothergill/Selwyn Taylor, "Some applications of a new electronic stethoscope", The Lancet 268 (1956) 1027-1028

Lacey, Kate, Listening Publics: The Politics and Experience of Listening in the Media Age (Cambridge 2013)

Lachmund, Jens, "Making sense of sound: auscultation and lung sound codification in nineteenth-century French and German medicine", Science, Technology, \& Human Values 24 (1999) 419-450

Lardner, Dionysus, The microscope: Excerpts from The museum of science and art (London 1854)

Phillips, A.F. "A loudspeaker for clinical teaching", British Heart Journal 11 (1949) $48-54$

Prentice, Rachel, "The anatomy of a surgical simulation: The mutual articulation of bodies in and through the machine", Social Studies of Science 35 (2005) 837-866

Rappaport, M.B./H.B. Sprague, "Physiologic and physical laws that govern auscultation and their clinical application", The American Heart Journal 21 (1941) 258-279

Rice, Tom, "'Beautiful Murmurs': Stethoscopic Listening and Acoustic Objectification", Senses and Society 3 (2008) 293-306

Rice, Tom, Hearing and the Hospital: Sound, Listening, Knowledge and Experience (Canon Pyon 2013)

Rice, Tom, "The hallmark of a doctor': the stethoscope and the making of medical identity", Journal of Material Culture 15 (2010) 287-301

Sterne, Jonathan, "Mediate auscultation, the stethoscope, and the 'autopsy of the living': Medicine's acoustic culture", Journal of Medical Humanities 22 (2001) 115-136

Sterne, Jonathan, "Headset Culture, audile technique, and sound space as private space", Journal for Media History 6 (2003) 52-82

Sterne, Jonathan, The Audible Past. Cultural Origins of Sound Reproduction (Durham 2003)

Swarup, Supreeya/Amgad N. Markaryus, "Digital Stethoscope: technology update", in: Medical devices: evidences and research (Dove Medical Press online 2018), URL: https://www.ncbi.nlm.nih.gov/pmc/articles/PMC5757962/pdf/mder-11-029. pdf

Tortil, Lauren, Une généalogie des grandes oreilles (Paris 2019)

Van Drie, Melissa, "Training the Auscultative Ear: Medical Textbooks and Teaching Tapes (1950-2010)", Senses and Society 8 (2013) 165-193

Van Drie, Melissa, "Hearing through the théâtrophone: sonically constructed spaces and embodied listening in late $19^{\text {th }}$ century French theatre", Sound Effects Journal: an interdisciplinary journal of sound and sonic experience (Special issue: Functional Sounds) 5 (2015) 73-90: https://www.soundeffects.dk/article/ view/23310

Van Drie, Melissa, "Refaçonner l'oreille, prendre en main la voix: le comédien et l'acteur", in: Julia Gros de Gasquet/Julie Valéro (eds), L'objet technique en scène. La mise en jeu des objets technologiques sur les scènes contemporains. Analyses et experiences (Paris 2019) 21-37

Winters, S.R., "Diagnosis by Wireless", Scientific American 124, June 11 (1921) 465 